\section{IL-17A AT CROSSROAD BETWEEN KERATINOCYTES AND FIBROBLASTS IN HUMAN SKIN WITHIN SYSTEMIC SCLEROSIS}

${ }^{1}$ AM Dufour*, 'M Alvarez, ${ }^{2} \mathrm{M}$-E Truchetet, ${ }^{3} \mathrm{NC}$ Brembilla, ${ }^{1} \mathrm{C}$ Chizzolini. ${ }^{1}$ Immunology and Allergy and Department of Pathology and Immunology, University Hospital and School of Medicine, Geneva, Switzerland; 'Rheumatology Department, Bordeaux University Hospital, Bordeaux, France; ${ }^{3}$ Dermatology and Department of Pathology and Immunology, University Hospital and School of Medicine, Geneva, Switzerland

\subsection{6/annrheumdis-2018-EWRR2018.19}

Introduction Increased levels of IL-17A have been reported in systemic sclerosis (SSc) but its role in fibrosis development is still debated. ${ }^{1}$ Recent findings suggest a role for keratinocytes in the development of fibrosis. ${ }^{2}$ Of interest, epithelial cells are preferential targets of IL-17A.

Objectives Our aim was to investigate the interactions between epidermis and dermis in the presence of IL-17A, taking into perspective the fibrotic process.

Methods Conditioned-media of primary human keratinocytes primed with IL-17A and/or TGF- $\beta$ were used to stimulate healthy donors (HD) and SSc fibroblasts. Alternatively, organotypic cultures of HD full human skin were treated with these cytokines. Responses were assessed by quantifying inflammatory mediators and type I collagen (Col-I) levels. The factors produced by keratinocytes were identified by a proteomic approach and their contribution was evaluated by their neutralisation. MicroRNA expression was examined by $\mu$ Paraflo technology platform.

Results Unstimulated HD- and SSc-derived keratinocyte-conditioned media (KCM) promoted collagen production by fibroblasts to a similar extent and in a dose-dependent manner. Cytokine array analysis and neutralising assays showed that TGF- $\beta$ was, at least in part, responsible for the pro-fibrotic effect of KCM. Although priming of keratinocytes with IL$17 \mathrm{~A}$ alone did not influence Col-I, it significantly decreased Col-I production induced by TGF- $\beta$ by fibroblasts $(p=0.02)$.

In full human skin, IL-17A promoted pro-inflammatory responses by inducing 2- to 4-fold increase of IL-8, IL-6, MCP-1 and MMP-1 levels, while showing direct anti-fibrotic effects and decreasing by 2 -fold collagen production triggered by TGF- $\beta \quad(p=0.02)$. The combined injection of IL-17A and TGF- $\beta$ in the full human skin resulted in a distinct pattern of miRNA expression, particularly driven by miR-4343, when compared to the expression induced by the separate injection of IL-17A and TGF- $\beta$.

Conclusions Keratinocytes profoundly influence dermal fibroblast responses, which are further modulated in the presence of IL-17A. These data support a role for keratinocytes in the pathogenesis of SSc. IL-17A acts as a potent anti-fibrotic factor in the model of keratinocyte - fibroblast interactions, as well as in the full human skin, which mechanisms are currently explored.

\section{REFERENCES}

1. Truchetet ME, et al. Arthritis \& Rheumatol 2013.

2. Takahashi T, et al. J Exp Med 2017.

Acknowledgements Work supported in part by grant 310030159999 from the SNF to CC.

Disclosure of interest None declared

\section{LONG NONCODING RNA H19X IS A MASTER REGULATOR OF EXTRACELLULAR MATRIX PRODUCTION IN SYSTEMIC SCLEROSIS}

${ }^{1} E$ Pachera*, ${ }^{2} S$ Assassi, ${ }^{2} G$ Salazar, ${ }^{1} M$ Frank-Bertoncelj, ${ }^{3}$ J Distler, ${ }^{1} G$ Kania, ${ }^{1} \mathrm{O}$ Distler. ${ }^{1}$ Center of Experimental Rheumatology, UNIVERSITY HOSPITAL ZURICH, Zurich, Switzerland; ${ }^{2}$ Department of Internal Medicine, University of Texas, Houston, USA; ${ }^{3}$ Department of Internal Medicine 3, University of Erlangen, Erlangen, Germany

\subsection{6/annrheumdis-2018-EWRR2018.20}

Introduction We have recently identified a novel, yet undescribed lncRNA, H19X, which was upregulated in the skin and lung of patients with SSc in a TGF $\beta$ dependent manner.

Objectives To characterise the function and the molecular mechanism of H19X in Systemic Sclerosis (SSc).

Methods The function of H19X was investigated in skin fibroblasts by knocking down H19X with locked nucleic acid oligonucleotides (LNA GapmeRs) and by using the following methods microarray analysis, immunofluorescence, Sircol, contraction assay, ELISA, Western blot (WB), in situ hybridization using Stellaris FISH probes and Assay for Transposase-Accessible Chromatin using sequencing (ATAC-seq).

Results Microarray analysis $(n=5)$ showed that after H19X silencing collagen catabolic process, extracellular matrix organisation and extracellular matrix disassembly were among the pathways with highest number of enriched genes. Sircol assay for pan-collagen production $(\mathrm{n}=5, \mathrm{p}<0.05)$, ELISA for procollagen $\mathrm{I} \alpha 1(\mathrm{n}=5, \mathrm{p}<0.05)$ and $\mathrm{WB}$ analysis for fibronectin $(n=7, p<0.05)$ confirmed the importance of H19X in the regulation of extracellular matrix components. Additionally, silencing of H19X significantly impaired $\alpha \mathrm{SMA}$ fibre formation, stress fibre formation as well as cell contractility strongly suggesting an important role of H19X in the development of the myofibroblast phenotype. Cell fractionation showed that TGF $\beta$ induced expression of H19X is localised mainly into the nucleus. In situ hybridization confirmed H19X localization as mainly nuclear and within a defined spot indicating that H19X could influence gene expression by interacting directly with the chromatin $(n=4)$. TFAP2a was identified as the transcription factor with the strongest difference of occupied binding sites after H19X downregulation.

Conclusions The novel lncRNA H19X appears to be a condition sine qua non for the profibrotic effects of TGF $\beta$. Mechanisms of action of the profibrotic effects of $\mathrm{H} 19 \mathrm{X}$ point to epigenetic regulation of the transcription factor. LncRNA open new perspectives in the pathogenesis of fibrotic diseases.

Disclosure of interest E. Pachera: None declared, S. Assassi: None declared, G. Salazar: None declared, M. Frank-Bertoncelj: None declared, J. Distler Grant/research support from: 4D Science, 1, Anamar, Active Biotech, Array Biopharma, BMS, Bayer Pharma, Boehringer Ingelheim, Celgene, GSK, Novartis, Sanofi-Aventis, UCB, 2, Actelion, Active Biotech, Anamar, Bayer Pharma, Boehringer Ingelheim, Celgene, Galapagos, GSK, Inventiva, JB Therapeutics, Medac, Pfizer, RuiYi and UCB, Consultant for: 4D Science, 1, Anamar, Active Biotech, Array Biopharma, BMS, Bayer Pharma, Boehringer Ingelheim, Celgene, GSK, Novartis, Sanofi-Aventis, UCB, 2, Actelion, Active Biotech, Anamar, Bayer Pharma, Boehringer Ingelheim, Celgene, Galapagos, GSK, Inventiva, JB Therapeutics, Medac, Pfizer, RuiYi and UCB, G. Kania Grant/research 
support from: Bayer, O. Distler Grant/research support from: Actelion, Bayer, BiogenIdec, Boehringer Ingelheim, Chemo$\mathrm{mAb}$, espeRare foundation, Genentech/Roche, GSK, Inventiva, Lilly, medac, MedImmune, Mitsubishi Tanabe Pharma, Pharmacyclics, Novartis, Pfizer, Sanofi, Sinoxa and UCB, 2, Actelion, Bayer, BiogenIdec, Boehringer Ingelheim, ChemomAb, espeRare foundation, Genentech/Roche, GSK, Inventiva, Lilly, medac, MedImmune, Mitsubishi Tanabe Pharma, Pharmacyclics, Novartis, Pfizer, Sanofi, Sinoxa and UCB

\section{MESENCHYMAL STEM CELL-DERIVED EXTRACELLULAR VESICLES: A NOVEL THERAPEUTIC OPTION IN SYSTEMIC SCLEROSIS}

${ }^{1} \mathrm{P}$ Rozier, ${ }^{1} \mathrm{M}$ Maumus, ${ }^{2} \mathrm{~A}$ Maria, ${ }^{3} \mathrm{~K}$ Toupet, ${ }^{4} \mathrm{C}$ Jorgensen, ${ }^{2} \mathrm{P}$ Guilpain, ${ }^{1} \mathrm{D}$ Noel ${ }^{*} .{ }^{1} \mathrm{Inserm}$; ${ }^{2}$ Médecine Interne et Maladies Multi-Organiques, CHU Montpellier; ${ }^{3}$ Montpellier University; ${ }^{4}$ Clinical immunology and osteoarticular diseases Therapeutic Unit, CHU Montpellier, Montpellier, France

\subsection{6/annrheumdis-2018-EWRR2018.21}

Introduction Systemic sclerosis (SSc) is a rare intractable autoimmune disease, with unmet medical need. Cell therapy using mesenchymal stem cells (MSC) is a promising approach, and we recently reported its efficacy in a murine model of SSc induced by hypochlorite $(\mathrm{HOCl})$. Since MSC act primarily through the secretion of soluble factors released within extracellular vesicles (EV), the use of EV instead of cells seems an attractive alternative. Herein, we compared the effects of two types of $\mathrm{EV}$, exosomes and microparticles, in $\mathrm{HOCl}$-induced SSc.

Objectives Herein, we compared the effects of two types of $\mathrm{EV}$, exosomes and microparticles, in HOCl-induced SSc.

Methods $\mathrm{BALB} / \mathrm{c}$ mice were challenged with daily intradermal $\mathrm{HOCl}$ injections during 6 weeks to induce SSc. Each group was treated at mid-experiment with infusions of $2.5 \times 10^{5}$ murine MSC, $250 \mathrm{ng}$ of exosomes or microparticles isolated from IFN $\gamma$-activated or non-activated (NA) MSC. We measured skin thickness every week. At euthanasia (d42), we analysed the expression of fibrotic and inflammatory markers (collagens 1 and 3, $\alpha$ Sma, TGF $\beta$, MMP 1 and 9, TIMP1, IL1 $\beta$, IL6, TNF $\alpha$ ) in lungs and skin samples using RT-qPCR.

Results Mice treated with each subtype of EV displayed lower clinical scores, less histological lesions, lower expression of fibrotic and inflammatory markers, with enhanced expression of remodelling parameters in skin and lung tissues. The observed effects were similar to those obtained with MSC. No difference was noted between NA and IFN $\gamma$-activated EV.

Conclusions MSC-derived EV display potent antifibrotic properties in murine SSc. This new acellular therapy represents a promising approach in this disease.

Disclosure of interest None declared
0022 FOR EACH HLA-DRB1 GENOTYPE, THE LIKELIHOOD TO DEVELOP RA CORRELATES WITH THE PROBABILITY OF BINDING AT LEAST A PEPTIDE FROM PAD4

${ }^{1,2} \mathrm{~N}$ Balandraud, ${ }^{2}$ Auger, ${ }^{3,4} \mathrm{G}$ Texier, ${ }^{1,5} \mathrm{~J}$ Roudier*. ${ }^{1}$ Rheumatology, Hôpital Sainte Marguerite; ${ }^{2}$ UMRs 1097 Immunogénétique des maladies auto immunes, INSERM and Aix Marseille University; ${ }^{3}$ Centre d'épidémiologie et de santé publique des armées (CESPA), CESPA; ${ }^{4}$ UMR 912 - SESSTIM, INSERM Aix Marseille University; ${ }^{5}$ UMRs 1097 Immunogénétique des maladies auto immunes, INSERM and Aix Marseille University, Marseille, France

\subsection{6/annrheumdis-2018-EWRR2018.22}

Introduction The production of IgG ACPA can be helped by $\mathrm{T}$ lymphocytes specific for PADs, the enzymes that transform arginin into citrullin. Thus, the molecular basis for the HLADRB1 association with RA might be the capability for the two alleles encoded by a given HLA-DRB1 genotype to bind PAD4 derived peptides. ${ }^{1}$ We recently published a table showing that the relative risk to develop ACPA positive RA for the 106 most common genotypes. ${ }^{2}$ French HLA-DRB1 genotypes varies from 28 to 0.2 . For a given HLA-DRB1 genotype, the risk to develop RA should be correlated with the probability for the two HLA-DRB1 molecules encoded by this genotype to bind peptides from PAD peptides.

Objectives To test whether the risk of developing ACPA positive RA for each HLA-DRB1 genotypes correlates with the probability for the 2 HLA-DR alleles encoded by each genotype to bind at least one PAD4 peptide.

Methods 65 Synthetic peptides (20 mers) of human PAD4 and 167 peptides encompassing the $\mathrm{A}$ and $\mathrm{B}$ chains of human fibrinogen were synthesised in solid phase. Whenever there was an arginine residue, both the arginine and the citrullin variant were synthesised. In the end, we had 25 fibrinogen peptides containing neither arginin nor citrulline, 71 citrullinated and 71 arginine peptides from fibrinogen.

HLA-DRB1 peptide binding studies were performed by adding one microgram of purified HLA-DRB1 to ELISA wells coated with 10 micrograms PAD peptide. Bound HLA-DR was revealed by biotinylated anti HLA-DR antibody followed by peroxidase conjugated avidin.

Statistical analyses Correlation between HLA-DRB1 genotypic risk for RA and Likelihood to bind PAD4 for a given genotype was evaluated by Spearman's

Results HLA-DRB1 genotypic risks to develop RA correlate with likelyhood to bind PAD4 peptides ( $p=0.06$, Pearson's), not citrullinated Fibrinogen peptides $(p>0.6$ and $p>0.9)$.

Conclusions HLA-DRB1 genotypes are associated with a risk to develop RA and a likelihood to bind at least one of 65 overlapping PAD4 peptides. The strong correlation between these two parameters suggest that PAD4 peptide binding to HLA-DRB1 may be the basis of the HLA-DRB1 RA association. Such correlation is not observed when testing the binding of citrullinated or native peptides from Fibrinogen to HLA-DRB1 molecules. 